\title{
El factor de impacto de la Revista Mexicana de Biodiversidad en el Science Citation Index para el 2007
}

\author{
Impact factor for the Revista Mexicana de Biodiversidad at the Science Citation Index in 2007
}

\author{
Layla Michán y Juan J. Morrone* \\ Museo de Zoología “Alfonso L. Herrera”, Departamento de Biología Evolutiva, Facultad de Ciencias, Universidad Nacional Autónoma de México. \\ Apartado postal 70-999, 04510 México, D.F., México. \\ *Correspondencia:jjm@hp.fciencias.unam.mx
}

\begin{abstract}
Resumen. La Revista Mexicana de Biodiversidad, previamente conocida como Anales del Instituto de Biología de la Universidad Nacional Autónoma de México, ha sido incorporada recientemente a la Web of Science. Su factor de impacto para el 2007 es 0.327, el cual es relativamente alto en relación con otras revistas latinoamericanas que publican artículos sobre biodiversidad.
\end{abstract}

\begin{abstract}
The Revista Mexicana de Biodiversidad, previously known as Anales del Instituto de Biología de la Universidad Nacional Autónoma de México, has been incorporated to the Web of Science. Its impact factor for 2007 is 0.327 , which results relatively high compared to other Latin American journals publishing articles on biodiversity.
\end{abstract}

La Revista Mexicana de Biodiversidad está entre las publicaciones periódicas latinoamericanas sobre biología de mayor antigüedad, tradición y relevancia. Fue fundada en 1930 y la edita el Instituto de Biología de la UNAM. En un principio se denominó Anales del Instituto de Biología de la Universidad Nacional Autónoma de México, serie Botánica (hasta 2005), serie Zoología (hasta 2005), serie Biología Experimental (hasta 1980) y serie Ciencias del Mar y Limnología (hasta 1974), títulos acordes con las líneas de investigación que se practicaban en cada departamento. En el 2005 la revista se renovó, fusionándose las series y cambiando el nombre, formato y estructura del Comité Editorial. En el transcurso del 2007, esta publicación periódica, después de un riguroso examen de acuerdo con los criterios de selección utilizados por el Science Citation Index (Thompson Reuters, 2008), fue incluida en la Web of Science, actualmente la base de datos bibliográfica, multidisciplinaria y de corriente principal de mayor prestigio.

A mediados del mes de junio de este año aparecieron los resultados del Journal Citation Report 2007, herramienta de Thomson Reuters que permite obtener algunos indicadores ciencimétricos de las revistas indizadas en la Web of Science. De estos indicadores, el factor de impacto (FI) resulta el más consultado por su aplicación en la evaluación científica. Éste se obtiene del cociente del total de citas obtenidas por cada revista durante el año al

Recibido: 26 junio 2008; aceptado: 26 septiembre 2008 que se refiere (2007) en documentos publicados los 2 años previos, entre el total de documentos publicados por esa misma revista durante 2005 y 2006. La Revista Mexicana de Biodiversidad en el 2005 publicó 22 artículos y en el 2006 fueron 33, la suma es 55. Durante el 2007 fueron citados 18 documentos, 4 (1 autocita) del 2006 y 14 (2 autocitas) del 2005. El factor de impacto así resultante fue de 0.327 .

La Revista Mexicana de Biodiversidad, de acuerdo con el criterio de clasificación temático que usa la Web of Science, pertenece a la categoría "Biodiversidad y Conservación". Ésta contiene 27 revistas, 2 de ellas de origen latinoamericano: la Revista Chilena de Historia Natural y la Revista Mexicana de Biodiversidad, que de acuerdo con su factor de impacto ocupan las posiciones 16 y 25, respectivamente. La de México ocupó el lugar 35 en relación a la totalidad de las revistas latinoamericanas y el quinto entre las mexicanas indizadas en el Journal Citation Report.

Existen otros cálculos disponibles en el Science Citation Index: el índice de inmediatez (las citas para los artículos publicados en el mismo año), que para la Revista Mexicana de Biodiversidad en el 2007 fue de 0. Las revistas más relacionadas se calculan con base en la fuerza de las relaciones entre las citas realizadas y obtenidas, esto es, la cantidad de citas de la revista en cuestión a la revista relacionada y las citas de la revista relacionada a la revista en cuestión. Las publicaciones periódicas más relacionadas con la Revista Mexicana de Biodiversidad 
fueron el Journal of Natural History y Zootaxa. Las revistas que citan artículos de la Revista Mexicana de Biodiversidad fueron 13 (incluyendo la propia revista), de las cuales 3 son latinoamericanas y 10 son extranjeras, lo que muestra el grado de internacionalización de la revista. A su vez, las revistas citadas por artículos de la Revista Mexicana de Biodiversidad fueron 243. Es de esperarse que en los próximos años estos indicadores mejoren, pues la inclusión de la revista en el SCI aumenta su difusión y las citas que recibe, como ha ocurrido en la mayoría de las revistas latinoamericanas sobre biodiversidad (en sentido amplio) incluidas en dicha base de datos (Cuadro 1).

Después de 1 año de su inclusión en la Web of Science, el impacto de la Revista Mexicana de Biodiversidad en la ciencia de corriente principal es alentador. $\mathrm{Su}$ factor de impacto subió de 0.267 en 2006 a 0.327 en 2007, esta última cifra fue mayor que otras revistas latinoamericanas de tradición y de mayor antigüedad presentes en el Science Citation Index (SCI) (Cuadro 1). Este FI de 2007 relativamente elevado, no refleja solamente el impacto alcanzado en un año, sino que seguramente hereda el reconocimiento adquirido por la publicación antecesora, los Anales del Instituto de Biología de la UNAM, lo que acumula una trayectoria e impacto que apenas hasta 2007 tiene expresión en el FI registrado por el Journal Citation
Report. Si bien el esfuerzo realizado es evidente, éste es sólo el principio, ya que habría varios retos a vencer. Entre ellos consideramos la modernización de los procesos de envío, la evaluación y seguimiento de los documentos sujetos a revisión en línea, la plataforma y el formato electrónico de la revista, acordes con los avances de nuestro tiempo. Por ejemplo, si se pudieran consultar los documentos en prensa, esto agilizaría su difusión. Si cada artículo se publicara en formato de blog, se aumentaría la discusión entre los especialistas, ya que cualquiera podría hacer comentarios en línea al documento, o incluso calificarlo. Estos recursos son algunos de los que están adoptando las publicaciones científicas electrónicas de la nueva generación. ¡Todavía hay mucho camino por recorrer!

Layla Michán agradece el apoyo del programa PROFIP-DGAPA, de la UNAM.

\section{Literatura citada}

Thomson Reuters. 2008. The Thomson Scientific Journal Selection Process. Consultado el 23 de junio del 2008 en: http://scientific.thomsonreuters.com/free/essays/ selectionofmaterial/journalselection/.

Cuadro 1. Factor de impacto en el SCI de las revistas relacionadas con biodiversidad publicadas en América Latina. El orden es descendente para el año 2007

\begin{tabular}{|c|c|c|c|c|c|c|c|c|}
\hline Revista & País & 2007 & 2006 & 2005 & 2004 & 2003 & 2002 & 2001 \\
\hline Memorias do Instituto Oswaldo Cruz & Brasil & 1.225 & 1.208 & 0.847 & 0.740 & 0.688 & 0.635 & 0.643 \\
\hline Anais da Academia Brasileira de Ciencias & Brasil & 0.895 & 0.737 & 0.653 & 0.435 & 0.510 & 0.469 & \\
\hline Revista Chilena de Historia Natural & Chile & 0.725 & 0.646 & 0.600 & 0.583 & 0.504 & 0.519 & 0.500 \\
\hline Revista Mexicana de Ciencias Geológicas & México & 0.586 & 0.682 & & & & & \\
\hline Ameghiniana & Argentina & 0.577 & 0.573 & 0.481 & 0.702 & 0.456 & 0.404 & 0.577 \\
\hline Revista Biología Marina y Oceanografía & Chile & 0.577 & & & & & & \\
\hline Revista Geológica del Chile & Chile & 0.567 & 1.147 & 0.615 & 0.912 & 0.536 & 0.630 & 0.321 \\
\hline Revista Brasileira de Entomologia & Brasil & 0.432 & & & & & & \\
\hline Revista Brasileira de Zoologia & Brasil & 0.422 & & & & & & \\
\hline Revista Brasileira de Zootecnia & Brasil & 0.388 & 0.385 & 0.250 & & & & \\
\hline Revista Mexicana de Biodiversidad & México & 0.327 & & & & & & \\
\hline Revista de Biología Tropical & Costa Rica & 0.274 & 0.217 & 0.322 & 0.22 & 0.354 & 0.107 & 0.097 \\
\hline Revista Fitotecnia Mexicana & México & 0.110 & & & & & & \\
\hline Revista Colombiana de Entomología & Colombia & 0.105 & & & & & & \\
\hline Revista Científica Facultad de Ciencias Veterinarias & Venezuela & 0.062 & 0.074 & 0.078 & 0.051 & 0.145 & 0.112 & 0.112 \\
\hline
\end{tabular}

